INTERNATIONAL DESIGN CONFERENCE - DESIGN 2018

https://doi.org/10.21278/idc.2018.0444

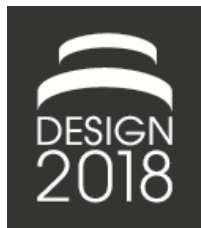

\title{
RE-DESIGN AND RE-MANUFACTURING OF DISCONTINUED SPARE PARTS IMPLEMENTING ADDITIVE MANUFACTURING IN THE MILITARY FIELD
}

\author{
J. Montero, K. Paetzold, M. Bleckmann and J. Holtmannspoetter
}

\begin{abstract}
In military systems, usually the repair of deployed equipment can become impossible due to the lack of a spare, either due to logistical problems or because said component is no longer in production. Thus, arises the need to manufacture the spare in the place where the equipment needs it, this gives an interesting opportunity to implement the techniques of additive manufacturing in the field. In this article a case study of a required spare of the German Federal Armed Forces is presented, with a methodology to deal with the data in the integrated process of design and manufacturing.
\end{abstract}

Keywords: reverse engineering, additive manufacturing, 3D printing, design process, digital design

\section{Introduction}

Capital-intensive goods, like aircrafts, commercial road vehicles and in some sense military equipment, are for a long period in service. In an advanced stage of their life cycle, the spare parts are no longer in production. As these equipment are dated from twenty or more years ago, the original manufacturing technology had not reached the degree of nowadays sophisticated technology level and during the detailed design stage did not include even the slightest consideration of the possibility to apply the techniques of additive manufacturing. In order to use new production technologies, like for instance additive manufacturing, digital 3D-data are required but usually not existent. Therefore, it is necessary to consider the technical documentation to generate 3D-data.

Particularly for military systems, due to the small lot size and the long production period (often with changes within the construction) the required technical documentation is not available. In this situation, the boundary conditions and the technical requirements of the product have to be evaluated. The process of reverse engineering becomes more difficult because the design department must create a new part without the knowledge of the boundary conditions for this part.

The American society for testing and materials (ASTM), created in 2009 the committee F42 in additive manufacturing (AM) and defines $\mathrm{AM}$ as the process of joining materials to make objects from threedimensional (3D) model data, usually layer by layer, as opposed to subtractive manufacturing methodologies (ASTM F2792-12a, 2012). Usually the term "3D printing" is used, due to the fact that by means of this process physical objects are created starting from digital files using a machine that instead of extracting material it adds material layer by layer. Currently, AM is widely used for rapid prototyping (RP) applications, since it is a very good tool to evaluate "how a product looks", for such applications the functionality of the created objects is limited or null, but with the latest advances in the improvement and understanding of properties and behaviour of the used materials AM is gaining 
popularity in the productive sector (Wohlers, 2017). At present there are many AM processes, and they are classified according to the material they use and the technology used for the process itself., the main AM technologies have been well-sorted by Huang et al. (2014). While there are advantages and disadvantages that all the AM processes share, there are those that do not, and that is what determines in the user the decision of which technology to use for each application. Our goal is the application of AM for the manufacture of spare parts in the military field, since it saves a lot of time in the logistics process, especially when the component is required overseas, and in particular when the component is no longer in production anymore, a an event that is normal when there are still relatively old machinery in operation. Some others actual advantages and disadvantages of AM are summarized in the work of Attaran (2017).

\section{State of the art}

In this document, we use the process of Selective Laser Melting (SLM) to produce metal parts and Fusion Deposition Modelling (FDM) to produce plastic parts. SLM is a technology that uses powder as a raw material, in this case Aluminium AlSi10Mg. In SLM the cross section of the 3D model data file is fully melted into a powder bed with a laser scanning system and forms the respective layer by its solidification, the first layer is built in a build platform, also called substrate, then a roller deposits a thin layer of powder on it, and the process repeat till all the layers are done (Bremen et al., 2012).

Figure 1a shows a schematic of this process. In the other hand, FDM is utilized to produce polymer parts, and uses a heating chamber to liquefy thermoplastic material that is fed into the system as a filament. This filament is pushed into the chamber by a tractor wheel mechanism and the fused material is extruded throughout a nozzle, being deposited in a build platform layer by layer till the completion of the desired object, a scheme of the process is shown in Figure 1b (Gibson et al., 2010).

a)

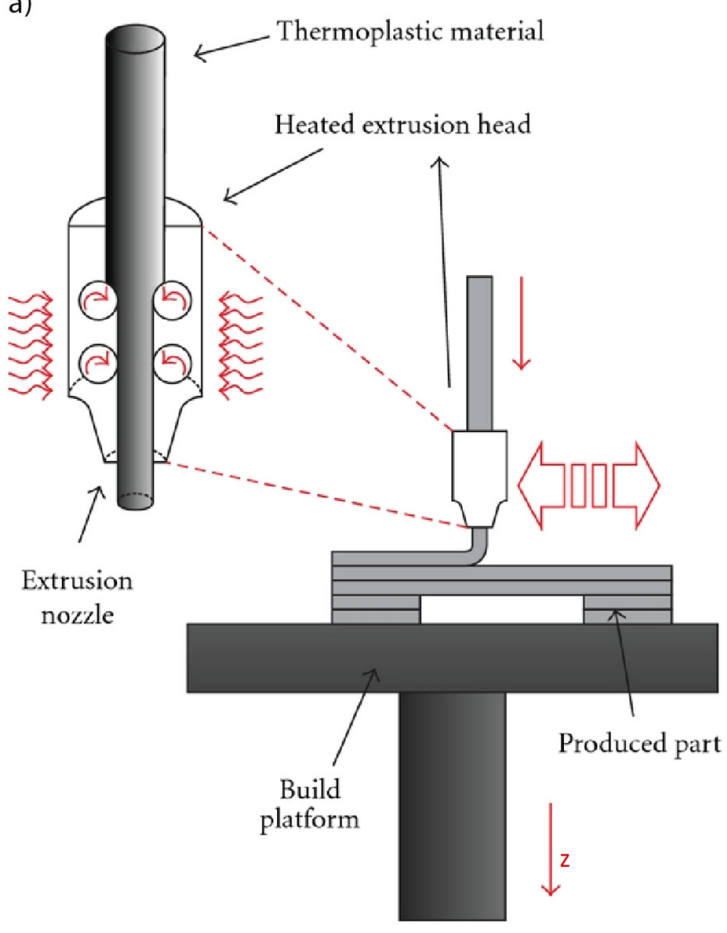

b)

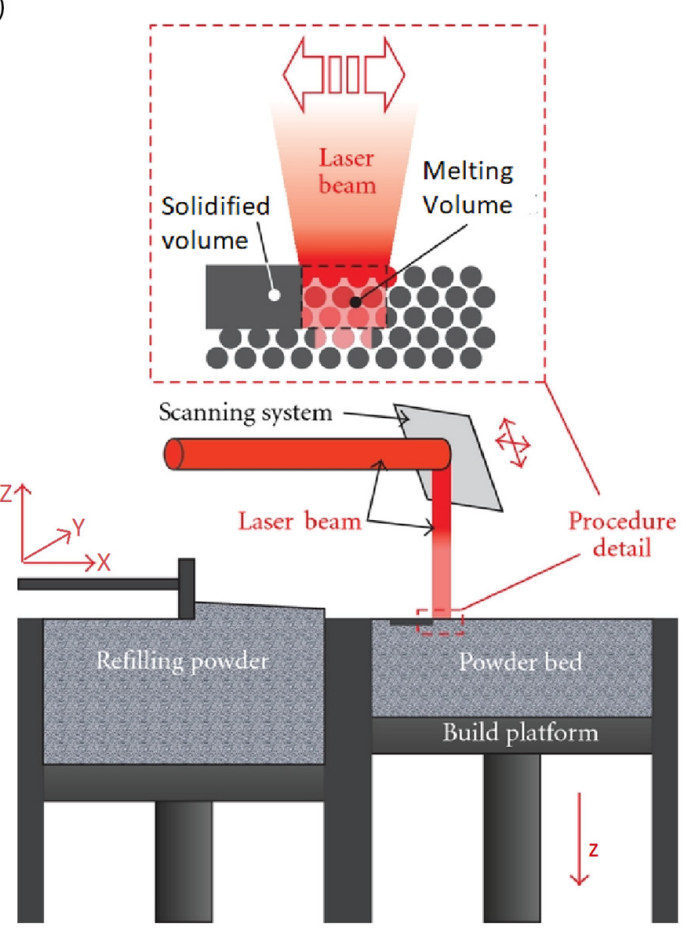

Figure 1. FDM technology (a) and SLM technology (b) schematic, Tsouknidas (2011)

Due to the development and commercial growth of the 3D laser scanning devices and many other 3D scanning technologies, the reconstruction of digital models from real physical objects has been facilitated during the last years (Gregor et al., 2008). This process is called reverse engineering (RE) 
in the field of computer aided design (CAD), (Varady and Martin, 2002), the goal of the RE is to replicate those features and geometric relations existing into an object starting from the acquired raw data from any $3 \mathrm{D}$ scanning technology. There is a lot of research on the subject RE, but few approaches integrate the geometric constraints on the part to obtain the features, which is what is necessary to obtain functional parts. The most well-known approaches are the "constrained fitting methods", this approach introduce geometric constraints by the formulation of an optimization problem (Benko et al., 2002), and the "beautification", which creates a fitting of the surfaces to build model and extract the features, relations and constraints from this reconstruction in a post-process stage (Langbein et al., 2004).

The process of RE is of utmost importance when it is sought to perform the replication of a spare efficiently, since the correct identification of the features depends on the functionality of the part at the end. But it must be borne in mind that as in this case, the part will then be created by AM, so some considerations must be taken into account when the user try to use the approach for working with AM. There is currently a lack of integrated approaches for RE processes focused on AM, since there are many types of features that can be improved or even eliminated keeping on mind the advantages attached to this type of manufacturing technology.

\section{Methodical framework}

\subsection{Case study: Valve cover}

Due to our field of interest about the application of new technologies such as additive manufacturing for the military industry, the component reviewed in this article was a request from overseas of the German armed forces. The case study is a valve cover used in multipurpose diesel electric generators, shown in Figure 2. This kind of spare part is usually a problem to get on the field, given that they are components made specifically for a certain utility where modular design is not suitable and as its break is very sporadic. Since these facts, is not usually to found it available in warehouse as well as other discontinued spare parts. All this added to the logistic process that involves moving the part around the different locations, if it is available, makes it a good case for its replication by additive manufacturing.

The equipment used for the scanning of this object was a Romer Absolute Arm with a Hexagon HP-L20.9 3D Laser Scanner. The equipment used for the manufacturing stage was an SLM125 Laser Melting machine for the replica in metal (AlSi10Mg) and a Stratasys Fortus $450 \mathrm{mc}$ fused deposition modelling machine for the replica in polymer (ABS).

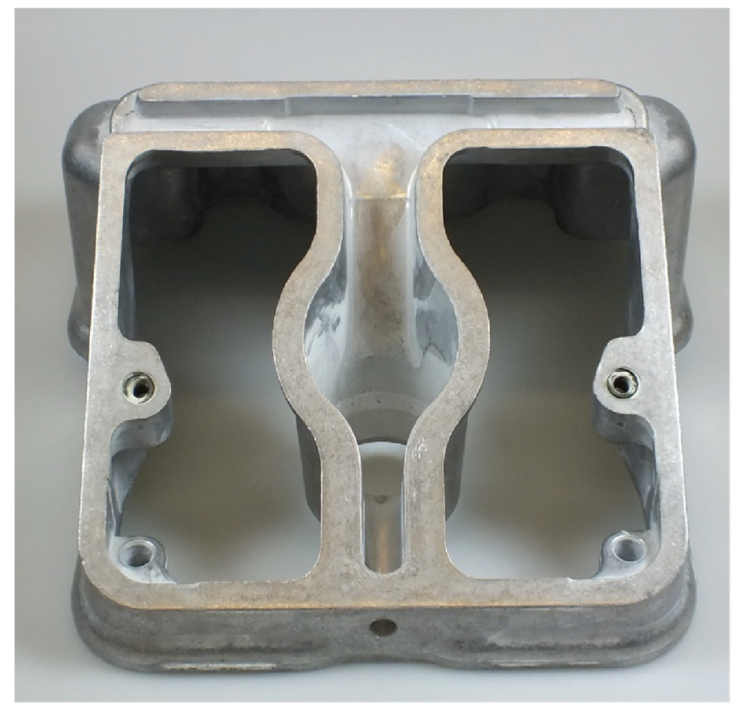

Figure 2. Valve cover used in electric diesel generators 


\subsection{Analysis of the process}

The entire reverse engineering process involves many well-differentiated stages in which the object to be replicated is analysed to obtain the design parameters that were used at the time of its manufacture (Venuvinod and Ma, 2004). Through this process, the new design is gaining characteristics that more and more resemble it to the original object (Klösch and Gall, 1995).

In our particular case we handle two ways to start with a design, the first and most natural is when the object to replicate is physically available and the second when it is not. These two starting point alternatives determine the nature of the input to our reverse engineering process. In the case of having the object to replicate (Input A), the input will be a cloud of coordinate points from a 3D laser scan, and in the case of not having the object (Input B); the input will be only some dimensions from the on-field measurements and the approximate shape of the part. Here it is easy to see that the better re-design can be done when the original object is available. While at first glance it seems an advantage to start from a 3D laser mapping of the object, this adds a sub-process to the stage of reverse engineering and here is where unwanted noise and distortion of the original features of the component are introduced, this is the reason why the smoothing and interpolation algorithms of the point cloud must be chosen carefully (Weyrich et al., 2004). It is also very common to obtain mappings with missing points in areas where the laser scanner cannot measure correctly; therefore, it is necessary to carry out a repair by software that joins surfaces with holes through a smoothing algorithm. All these issues added to the inaccuracy of the laser when performing the work and external factors such as vibration or lack of reflectivity that may have existed during the scan, make the point cloud is not an exact digital copy of the component. Finally, when a physical disposing of the object is not possible, the design department must work even more because they will build a component respecting its dimensions and those boundary conditions and environmental conditions that may exist, so the design is more objective, but no easier. In this document, the first option is analysed in depth, which in the military field is very usual when spare parts are needed overseas and are neither longer manufactured nor available.

The final goal of this stage is to obtain the technical drawings of the component in a CAD file, which is then converted to an STL file that is a non-parameterized point cloud, the latter is the final input to the software that handles the additive manufacturing machine. The STL file is finally sliced in layers and a support structure is added before "sending to print", this step is very well implemented automatically for each proprietary software of each AM machine according to its capabilities. It is for this reason that the work of the design department ends with the delivery of the STL file that is, after all, the digitized product; from this moment, it is the task of the manufacturing department to physically deliver the component. An overview of the overall process can be observed in Figure 3.

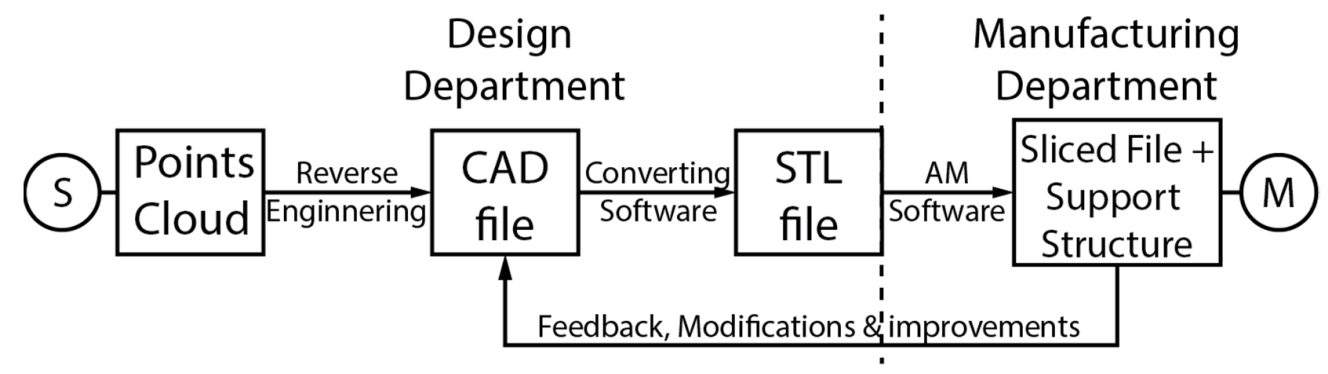

Figure 3. Evolution of the data from scanning(S) to manufacturing(M)

The determining factor in the work of the design department is the one that transforms the cloud of points in the CAD drawings, here it is necessary the precise identification of the features of the part and even more important to know which ones are important and which are not. From this process will depend the time and resources invested, because there are many features in components that are created appropriate to the manufacturing method that was used to manufacture the original part, an example are the extraction supports for castings or the access holes for machining of interior surfaces, which when the same component is produced by AM are neither necessary nor useful. For this reason, the reverse engineering stage for AM requires a deeper analysis based on the available tools and how to perform a progression of the data flow to obtain reliable results. 


\subsection{Data flow on the toolchain of the design department}

When a component is left into the design department, the scan and first digitization of the model is carried out, here, a file with a point cloud is obtained, depending the size on the resolution of the 3D scanner in use. The denser this point cloud, the better it will be the quality of the file, and easier to carry with the completion of the subsequent steps. After that ends what we call Digitalization Stage 1(DS1). Normally, the result of the DS1 has gaps or discontinuities as well as inaccuracies typical of the type of scanner implemented, for this reason in the DS2 this point cloud is repaired by software, smoothing algorithms are used and the zones that the designer identifies as erroneous or discontinuous when compared with the original object are modified. Once the point cloud is repaired, the process of recognizing the features and geometric relations that it contains is realized, sub-process DS3, this is the core of the reverse engineering process. In the DS3 the greatest difficulties are encountered, if well it is possible to apply those templates-based reverse engineering approaches when the number of features is considerable high they have not been entirely useful. Therefore, it is necessary to depend of the skill of the designer to know what are the correct features and how the geometric relations should be respected. While many types of features can be identified, there are many that do not have utility for the functionality of the component, that is, in an ideal world each requirement has a feature associated with it and the sum of these features forms the part, but as the parts must adjust the manufacturing method for which they were designed is normal the existence of features that have associated non-functional requirements such as for example can be an extraction support for castings. This process is outlined in Figure 4, and the first CAD file that will be subject to modifications in the following sub-processes is delivered from this stage.

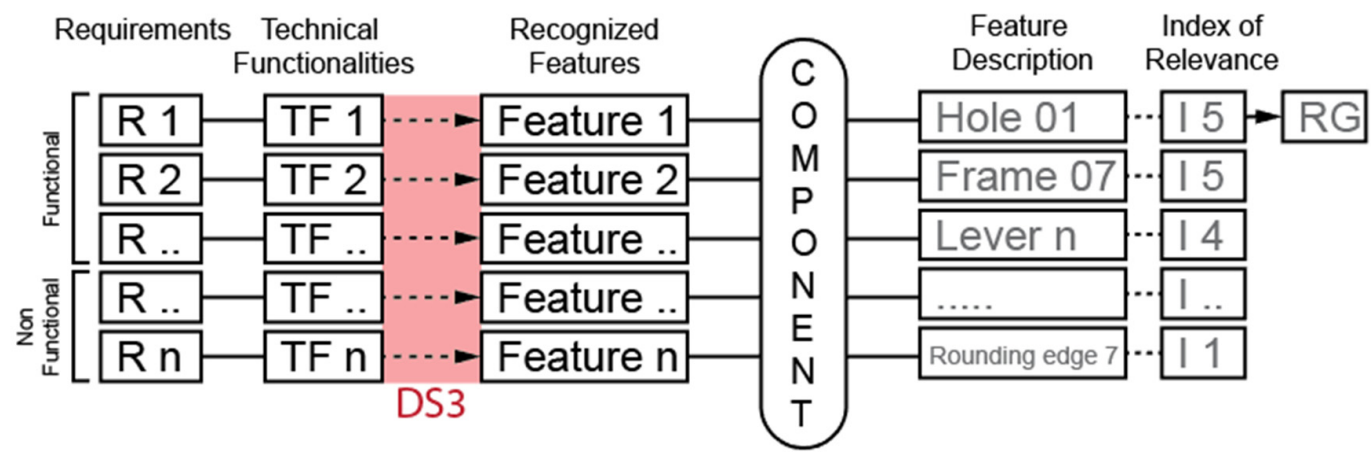

Figure 4. Design stage 3 outline

Taking into account this last consideration another critical stage appears, the sub-process DS4, which is the decision that the designer must take to eliminate those features that are not functional and does not compromise the integrity of the component. This can be done because when a part is produced with AM since there are no restrictions of shape or form regarding the manufacturing method. Exists also the situation in which features must be added to meet certain requirements unreachable for AM, such as the case of mechanized surface finishing, generally this can be solved by adding material thinking in advance of a post-milling stage. In this sub-process, it is also possible to carry out an optimization of the part, applying the well-known topological optimizations (Osanov and Guest, 2017), form optimizations (Lee, 2007) or size optimizations (Soni et al., 2016), these pursue an improvement in performance by optimizing the mechanical properties of the component.

As a last resort the component is verified in the sub-process DS5, by checking the boundary conditions that must be fulfilled and if the geometrical restrictions respect those of the original model. Additionally, a simulation using finite element method (FEM) software is an option to have a better certainty that the part will meet the requirements satisfactorily. At the end of this step, the final product of the design department is delivered, which is the STL file with the part to be manufactured by the manufacturing department. A visualization of how the design department deal with this whole process and how the data flows through the reverse engineering process for AM is shown in Figure 5 for the two types of data input mentioned in the previous section. 


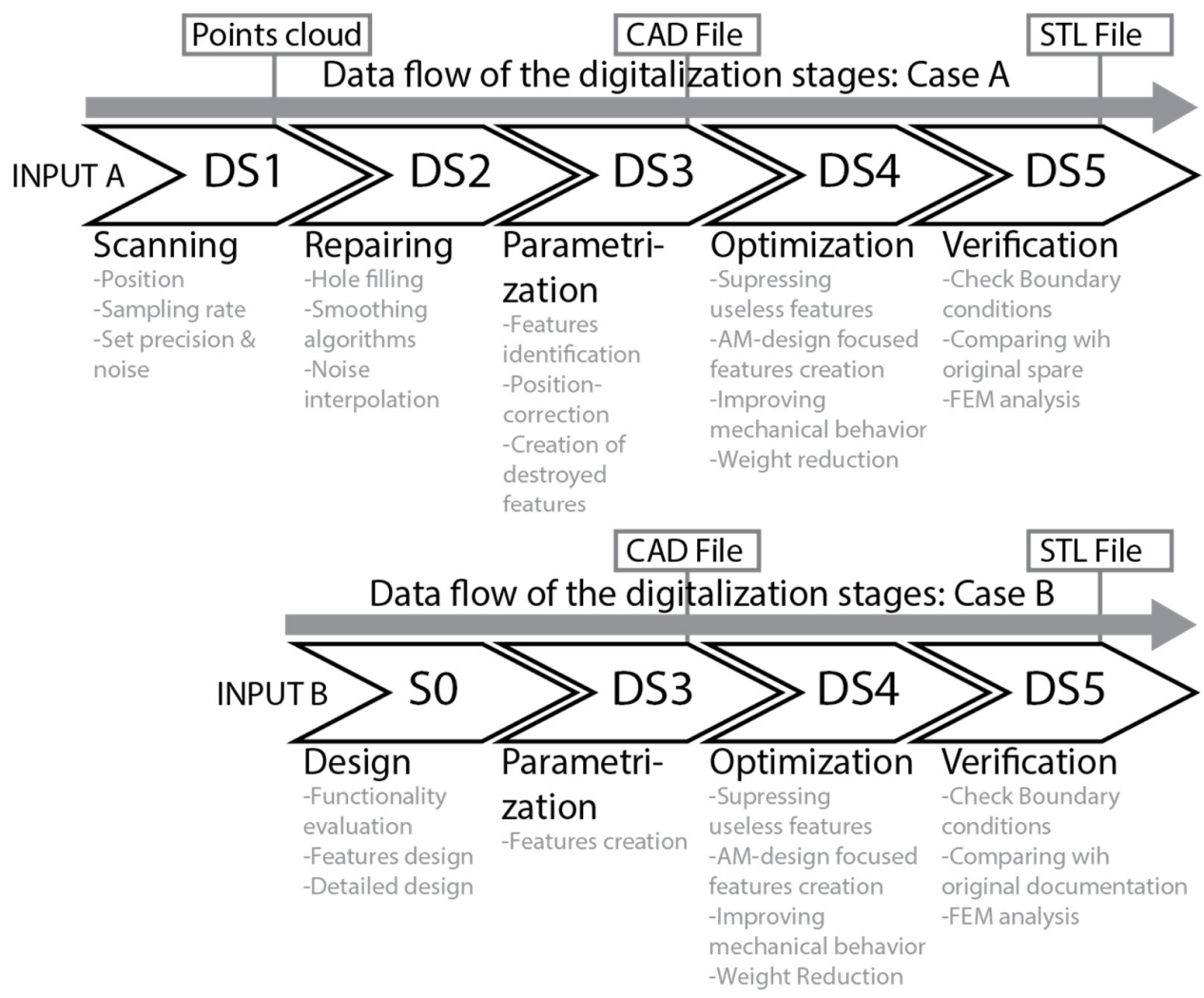

Figure 5. Activities through the digitalization stages on the design department

It is important to bear in mind that some of the modifications that could be made in the DS4 may not verify the DS5 stage and then it is necessary to modify the original CAD file to adjust the component to the new parameters. When the final STL file is delivered, the manufacturing department adds the support structures the part will need and also verifies that the construction is viable, there are times when the support structures cannot be generated automatically by the software handling the machine, and in that case the design department is the one who must also take charge of the design of the support structure. As it can be seen in Figure 6, this generates a feedback in the design-manufacturing process and converts it into a closed circuit, where the integration of both parts is necessary to achieve the effective realization of components with AM.

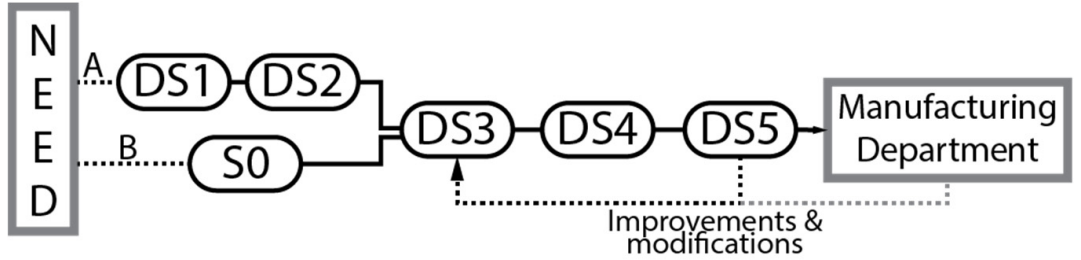

Figure 6. Overview of the RE design-manufacturing process

\subsection{Parametrization stage}

Given that the most critical part of the toolchain is the DS3, it deserves a differentiated analysis from the rest of the DS integrating the dataflow. We divided the study of the stage taking into account considerations focused on the used AM and separately in the process of recognition and design of features.

\subsubsection{Geometry and constraints considerations}

In order to optimize the process and facilitate the understanding of the operation of the component in the next stage DS4, the recognized features are organized with a relevance index from I1 to I5. Where 
I5 represents that the identified feature is extremely important for the functionality of the part and I1 represents that the feature is not relevant or simply fulfils a cosmetic function, as shown in Figure 4. This index allows the designer to be clear about the geometrical characteristics that will govern the shape of the component, since they will be used as constraints to make the CAD model. Those most important features are used as reference geometry (RG), and from one of these, with the choice based on the criterion of the designer, will be set the origin of the CAD model. Knowing how to choose a RG is a very important process because then in future modifications, the dimensions of the part will be subject to them. As an example, in a simple component such as a bolt the RG will be the circle that generates the main cylinder and the zero will be conveniently located at the centre of the circle. In the case of the valve cover, the chosen geometry reference was a fixing hole corresponding to a screw that links the component with the electrical generator's engine block.

Due to the fact that the dimensional analysis comes from a used component, it is probable that many features are dimensionally poorly identified due to wearing. Here again comes the criterion of the designer to understand that certain components subject to wear show different dimensions than the original ones as is the case of holes housing moving components or edges exposed to abrasive contact. In our case study was necessary to modify non-linear edges, threaded holes that did not respond to a standard thread, non-circular holes, contact surfaces for gaskets that were not flat. As a result of this process it is obtained a solid CAD model with no abrasion or damages, unlike what usually is sent to the manufacturing department coming from a simply scanned file with no data post-processing.

\subsubsection{Manufacturing method considerations}

For this case study the chosen manufacturing method is SLM with the ALSi10Mg material, the machine to be used is an SLM 125 equipped with a $400 \mathrm{~W}$ fiber laser and a laser beam diameter of $70 \mu \mathrm{m}$. Because it is well known that the SLM process leaves high values of residual stresses in the components (Brandl et al., 2012), it is taken into account that at the end of the manufacturing process a post-treatment of stress-relieving will be carried out $2 \mathrm{hs}$ at $250{ }^{\circ} \mathrm{C}$ accordingly to Mertens et al. (2015).

Over the last few years, extensive studies have been carried out on the mechanical properties and microstructure of AlSi10Mg, which have shown that properties such as hardness, ultimate tensile strength, elongation and impact energy are comparable to the AlSi10Mg casted material, due to the fine distribution of the phase Si in the microstructure (Aboulkhair et al., 2016). It is no less important to take into account a greater consideration and is that often the components show anisotropy to the elongation at break in $\mathrm{Z}$ direction according to Figure 1, (Kempen et al., 2012). This is the reason why the direction with foreseeable major properties is perpendicular to the laser beam i.e. the XY plane.

This latter information is taken into account in the DS3 because while the parameterization of the part is carried out, the critical sections are made to coincide with the planned manufacturing direction in accordance with the XY plane whenever possible. When a part presents structural problems and it is decided to make an improvement, the reinforcements are designed to be requested preferably in the XY plane.

Another relevant factor is to involve a support structure into the model. There are complex cases in which the support structure is very necessary to carry out the manufacturing stage and there are cases in which it is practically not needed. Although the use of these support structures generates effects on the final distortions of the part shape, this has been significantly reduced by controlling the temperature during manufacturing (Buchbinder et al., 2014), that is a huge advance because it helps to reduce the workload in the DS3, and therefore its influence is not taken into account, unless it is required from a previous iteration, (feedback from the DS5 or from the manufacturing department).

After the parameterization of the part, the aforementioned considerations influence the recommended manufacturing position where the correspondence of the directions with better properties and the critical sections of the part is prioritized, and in the second place the minimization of the support structure.

\section{Results and discussion}

For the DS1, the scan of the valve cover was carried out with a Hexagon RS4 3D Laser scanner coupled to a metrology arm Romer Absolut. Since the surface of the aluminum component was reflective, a thin layer of anti-reflective spray was applied because reflective surfaces cannot be correctly scanned. The post-process of the points cloud in the DS2 was made using the Innovmetric Polyworks software, the gaps 
of the zones that have not been properly scanned were filled and those surfaces in which the point cloud was irregular were smoothed. In the DS3, the main features were recognized using the Polyworks Inspector software and the dimensions of interest were extracted. The parameterized model was created using the Solidworks software, using as a basis the recognized features and reference measurements. Here the RE approach "constraint-fitting" was used, because it is more suitable for this type of components, since the fact of starting from the relationships that the designer considers fundamental (such as the parallelism between two faces belonging to a wall or the symmetry with respect to the geometric centre of the fixing holes) is advantageous for obtaining at the end relations that are more consistent and easy to modify for the component without ruining the rest of the model. In addition, as this component is part of an existing set, it must fit perfectly, so the boundary conditions of the valve cover were therefore used also as constraints. The symmetry of the model was used and only RE was made on the half of the valve cover.

In the DS4, those features that were not considered necessary for the functionality of the component were eliminated, in order to save material and manufacturing time, an example of this is the tabs that are used to extract the part when it is cast in the original mold. It remains as a future project apply the use of the site-specific properties described by Tammas-Williams and Todd (2017), in the sections where the thickness of the part allows it. During the DS5, the measurements of the new design were verified and compared with the points cloud obtained in the DS1 using the Polyworks software, obtaining a maximum deviation of the order of $0.1 \mathrm{~mm}$ in areas the designer did not consider relevant. A no less important fact was that the position of the part in the STL file was not the propitious to print the component with the automatic support structure generator, then remitted to adjust the position in the DS3 and finally delivered to the department of manufacture. In Figure 7 it can be seen the evolution of the component in the design department and in Figure 8 the manufactured part as it comes out from the SLM machine on its build platform.
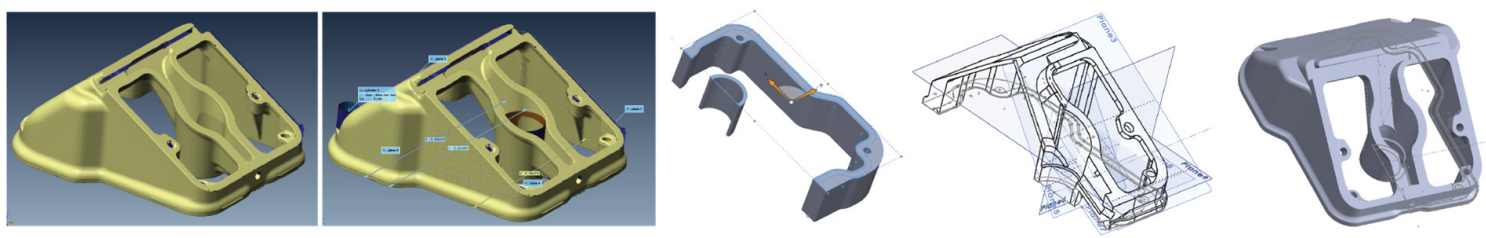

Figure 7. Evolution of the component through the digitalization stages

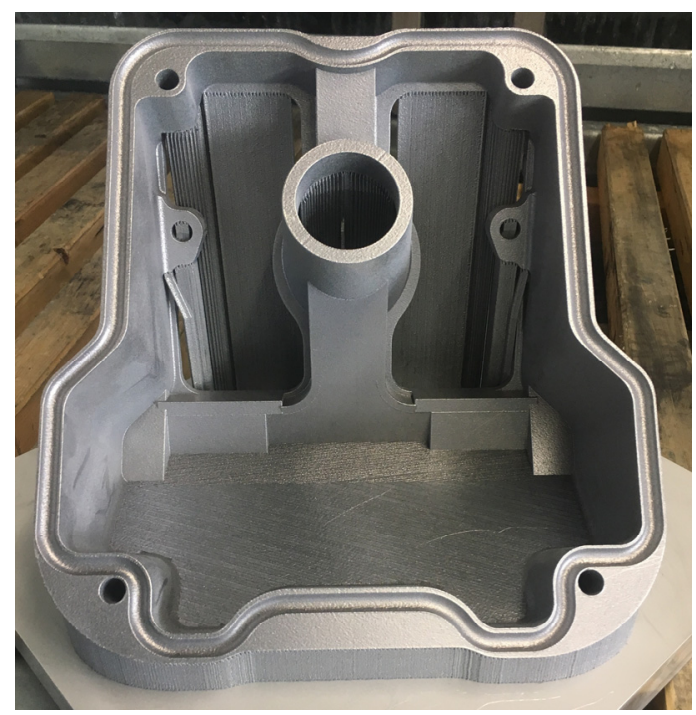

Figure 8. Re-manufactured component

As a discussion, it can be mentioned that during the recognition of features in the DS3, mistakes can be made and the recognized features could not correspond with those originals of the part as shown in the diagram of Figure 9, where the elements surrounded with a dashed line are unknown. An example is 
when the recognized feature does not actually exist in the original model and therefore does not have any associated Technical Functionality (Feature 3 in Figure 9). It is also possible that a Technical Functionality is known and as the associated feature could not be recognized (Feature 5 in Figure 9), then it must be reconstructed, as is the case in which the scanned part has some missing, broken or worn feature. As a last variant, it may be that a feature is recognized but is not possible to associate with any Technical Functionality (Feature 2 in Figure 9), and therefore the designer cannot evaluate its importance in the operation of the component, and its possible elimination or improvement.

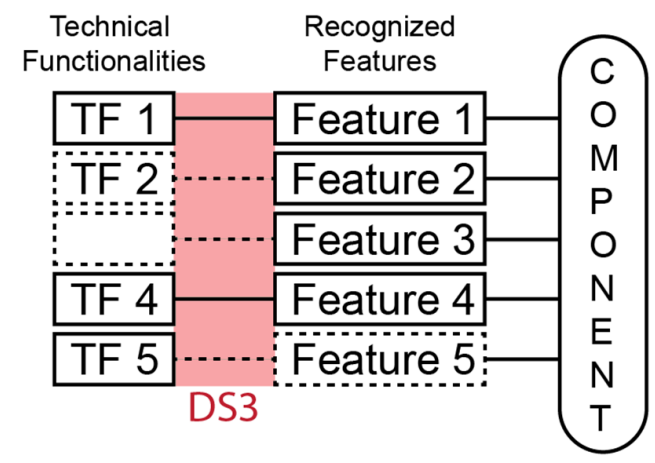

Figure 9. Variant of the DS3 outline

\section{Conclusion}

While there are many automatic tools to facilitate the process of reverse engineering, to this day is still very important the vision of the designer, since at the end of the day is the one having the criteria to make decisions that gives the final shape to the component.

Organizing the reverse engineering process adjusted for AM in the design room in different digitalization stages, has given us a good perspective to find which are the critical points on which more efforts should be concentrated. It is feasible to consider the DS3 as the most important and critical stage of the integrated process, given that it is where the designer makes his biggest contribution, due to his experience and without losing sight of the feedback coming from the later stages. That is why arises the need of creating a set of well-defined rules that must be met in the design department when adjusting or modifying a Spare for AM, especially in the military field where designers can be deployed overseas working in a team formed by temporary members, and therefore the advances must be well documented so that the accumulation of experience is not lost.

It should be noted that the integrated design process for AM, if it is well done, must be done only once, then when the same spare is required, only the STL file is sent to the manufacturing department.

It is clear that AM is very useful for manufacturing functional spare parts if the correct methodology is applied, and that is the reason why today there is so much interest in it, especially in those situations where the logistics process is hindered to deliver new spare parts or when spare parts are no longer in production.

\section{References}

Aboulkhair, N.T., Maskery, I., Tuck, C., Ashcroft, I. and Everitt, N.M. (2016), "On the formation of AlSi10Mg single tracks and layers in selective laser melting: Microstructure and nano-mechanical properties", Journal of Materials Processing Technology, Vol. 230, pp. 88-98. https://doi.org/10.1016/j.jmatprotec.2015.11.016

ASTM F2792-12a (2012), Standard Terminology for Additive Manufacturing Technologies (Withdrawn 2015), ASTM International, West Conshohocken, PA. https://doi.org/10.1520/F2792-12A

Attaran, M. (2017), "The rise of 3-D printing: The advantages of additive manufacturing over traditional manufacturing”, Business Horizons, Vol. 60 No. 5, pp. 677-688. https://doi.org/10.1016/j.bushor.2017.05.011

Benko, P., Kós, G., Várady, T., Andor, L. and Martin, R. (2002), "Constrained fitting in reverse engineering", Computer Aided Geometric Design, Vol. 19 No. 3, pp. 173-205. https://doi.org/10.1016/s01678396(01)00085-1

Brandl, E., Heckenberger, U., Holzinger, V. and Buchbinder, D. (2012), “Additive manufactured AlSi10Mg samples using Selective Laser Melting (SLM): Microstructure, high cycle fatigue, and fracture behavior", Materials \& Design, Vol. 34, pp. 159-169. https://doi.org/10.1016/j.matdes.2011.07.067 
Bremen, S., Meiners, W. and Diatlov, A. (2012), “Selective Laser Melting”, Laser Technik Journal, Vol. 9 No. 2 , pp. 33-38. https://doi.org/10.1002/latj.201290018

Buchbinder, D., Meiners, W., Pirch, N. and Wissenbach, K. (2014), "Investigation on reducing distortion by preheating during manufacture of aluminum components using selective laser melting", Journal of Laser Applications, Vol. 26 No. 1, pp. 012004. https://doi.org/10.2351/1.4828755

Gibson, I., Rosen, D.W. and Stucker, B. (2010), "Printing Processes”, In: Gibson, I., Rosen, D.W. and Stucker, B. (Eds.), Additive Manufacturing Technologies - Rapid Prototyping to Direct Digital Manufacturing, Springer, Boston, pp. 187-222. https://doi.org/10.1007/978-1-4419-1120-9_7

Gregor, M., Budzel, F., Stefanik, A. and Plinta, D. (2008), "3D Laser Scanning in Digitization of Current Production Systems", IFAC Proceedings Volumes, Vol. 41 No. 3, pp. 86-93. https://doi.org/10.3182/20081205-2-cl-4009.00017

Huang, Y., Leu, M.C., Mazumder, J. and Donmez, A. (2014), “Additive Manufacturing: Current State, Future Potential, Gaps and Needs, and Recommendations", Journal of Manufacturing and Engineering, Vol. 137 No. 1, pp. 014001. https://doi.org/10.1115/1.4028725

Kempen, K., Thijs, L., van Humbeeck, J. and Kruth, J.-P. (2012), "Mechanical Properties of AlSi10Mg Produced by Selective Laser Melting”, Physics Procedia, Vol. 39, pp. 439-446. https://doi.org/10.1016/j.phpro.2012.10.059

Klösch, R. and Gall, H. (1995), "Reverse Engineering”, In: Klösch, R. and Gall, H. (Eds.), Objektorientiertes Reverse Engineering, Springer, Berlin, Heidelberg, pp. 1-40. https://doi.org/10.1007/978-3-642-79221-2_1

Langbein, F.C., Marshall, A.D. and Martin, R.R. (2004), "Choosing consistent constraints for beautification of reverse engineered geometric models", Computer-Aided Design, Vol. 36 No. 3, pp. 261-278. https://doi.org/10.1016/s0010-4485(03)00108-8

Lee, T.H. (2007), “Shape Optimization”, In: Arora, J.S. (Ed.), Optimization of Structural and Mechanical Systems, World Scientific, pp. 149-159. https://doi.org/10.1142/9789812779670_0005

Mertens, A., Dedry, O., Reuter, D., Rigo, O. and Lecomte-Beckers, J. (2015), "Thermal Treatments of A1Si10Mg Processed by Laser Beam Melting", Proceedings of the 26th International Solid Freeform Fabrication Symposium, Austin, Texas, August 10-12, 2015, pp. 1007-1016.

Osanov, M. and Guest, J.K. (2017), "Topology Optimization for Additive Manufacturing Considering LayerBased Minimum Feature Sizes”, Proceedings of ASME 2017 International Design Engineering Technical Conferences and Computers and Information in Engineering Conference, Vol. 2A, Cleveland, Ohio, August 69, 2017, ASME, pp. V02AT03A036. https://doi.org/10.1115/DETC2017-68383

Soni, N., Dave, P., Patel, V. and Savsani, V. (2016), "Size and shape optimization of structures using GA-FEA interface", International Journal of Technology and Engineering, Vol. 2 No. 3, pp. 76-82. https://dx.doi.org/10.20469/ijtes.2.40003-3

Tammas-Williams, S. and Todd, I. (2017), "Design for additive manufacturing with site-specific properties in metals and alloys", Scripta Materialia, Vol. 135, pp. 105-110. https://doi.org/10.1016/j.scriptamat.2016.10.030

Tsouknidas, A. (2011), "Friction Induced Wear of Rapid Prototyping Generated Materials: A Review", Advances in Tribology, Vol. 2011, pp. 1-7. https://doi.org/10.1155/2011/746270

Varady, T. and Martin, R. (2002), "Reverse Engineering”, In: Farin, G., Hoschek, J. and Kim, M.-S. (Eds.), Handbook of Computer Aided Geometric Design, Elsevier, pp. 651-681. https://doi.org/10.1016/b978044451104-1/50027-7

Venuvinod, P.K. and Ma, W. (2004), "Reverse Engineering and CAD Modeling”, In: Venuvinod, P.K. and Ma, W. (Eds.), Rapid Prototyping - Laser-based and Other Technologies, Springer, Boston, MA, pp. 75-134. https://doi.org/10.1007/978-1-4757-6361-4_4

Weyrich, T., Pauly, M., Keiser, M., Heinzle, S., Scandella, S. and Gross, M. (2004), "Post-processing of scanned 3D surface data", Proceedings of SPBG'04 / the First Eurographics conference on Point-Based Graphics, The Eurographics Association, pp. 85-94. https://doi.org/10.2312/SPBG/SPBG04/085-094

Wohlers, T.T. (2017), Wohlers Report - 3d printing and additive manufacturing state of the industry, Wohlers Associates, Fort Collins.

Joaquin Montero, M. Sc. in Aerospace Engineering

Bundeswehr University Munich, Institute for Technical Product Development

Werner Heisenberg Weg 39, 85577 Neubiberg, Germany

Email: j.montero@unibw.de 